\title{
Analysis of Waterman's Method in the Case of Layered Scatterers
}

\author{
Victor Farafonov, ${ }^{1}$ Vladimir Il'in, ${ }^{1,2,3}$ Vladimir Ustimov, ${ }^{1}$ and Evgeny Volkov ${ }^{2}$ \\ ${ }^{1}$ St. Petersburg State University of Aerospace Instrumentation, Bol. Morskaya 67, St. Petersburg 190000, Russia \\ ${ }^{2}$ St. Petersburg University, Universitetsky Pr. 28, St. Petersburg 198504, Russia \\ ${ }^{3}$ Pulkovo observatory, Pulkovskoe Sh. 65/1, St. Petersburg 196140, Russia
}

Correspondence should be addressed to Vladimir Il'in; v.b.ilin@spbu.ru

Received 6 September 2016; Accepted 21 December 2016; Published 16 January 2017

Academic Editor: Antonio Scarfone

Copyright (C) 2017 Victor Farafonov et al. This is an open access article distributed under the Creative Commons Attribution License, which permits unrestricted use, distribution, and reproduction in any medium, provided the original work is properly cited.

\begin{abstract}
The method suggested by Waterman has been widely used in the last years to solve various light scattering problems. We analyze the mathematical foundations of this method when it is applied to layered nonspherical (axisymmetric) particles in the electrostatic case. We formulate the conditions under which Waterman's method is applicable, that is, when it gives an infinite system of linear algebraic equations relative to the unknown coefficients of the field expansions which is solvable (i.e., the inverse matrix exists) and solutions of the truncated systems used in calculations converge to the solution of the infinite system. The conditions obtained are shown to agree with results of numerical computations. Keeping in mind the strong similarity of the electrostatic and light scattering cases and the agreement of our conclusions with the numerical calculations available for homogeneous and layered scatterers, we suggest that our results are valid for light scattering as well.
\end{abstract}

\section{Introduction}

Scattering of light by small particles is an essential part of various applications in physics of atmosphere, ecology, biophysics, astrophysics, and many other fields. The problem of light scattering is frequently treated by a method suggested by Waterman $[1,2]$. In this method, the fields are expanded in terms of wave functions and the field expansions are substituted in the surface integral equations equivalent to the Helmholtz equation with the standard boundary conditions. Linear independence of the wave functions allows one to get an infinite system of linear algebraic equations relative to the unknown field expansion coefficients. In numerical calculations, the system is truncated, and often the relation between the unknown and known expansion coefficients formulated in the matrix form is described by the transition $T$-matrix (see for more details [3]). The method has become very popular (see [4] and references therein) and got several nearly equivalent names: the extended boundary condition method, the $T$-matrix method, and the null-field method, depending on the accents being made (see, e.g., $[5,6])$.
Applicability of this method has been considered in the analytical and numerical ways. The results derived were controversial. For instance, calculations demonstrated serious computational problems for spheroids with the aspect ratio $a / b$ above $\sim 10$ (the use of extended precision reduces these problems but does not remove them [7]), while theories either said that the approach should have no limits [8] or concluded that singularities of the wave fields led to method inapplicability to spheroids with, for example, $a / b>\sqrt{2}$ [9]. In the meantime, a recent improvement of the numerical implementation of the method in [10] has demonstrated that the method should not have any limitations for spheroids. A comparison of the theoretical studies showed that their main difference is related to an unclear status of singularities of the analytic continuation of the internal field. An up-to-date review of the works on the subject can be found in [11].

The state-of-the-art analysis has been presented in [11], where in the electrostatic case it is shown that the method is applicable to homogeneous (nonspheroidal) scatterers; that is, it gives the infinite system relative to the unknown field coefficients which is solvable and solutions of the truncated 
systems converge to the solution of the infinite system, under the condition that all singularities of the internal field are more distant than all singularities of the scattered field. Due to a clear similarity of the electrostatic and light scattering cases (see, e.g., [5]), one can expect that the results of [11] are applicable to light scattering.

It should be emphasized that Waterman's method has been thoroughly analyzed just for homogeneous particles, while many natural scatterers are inhomogeneous, with two nearly alternative often utilized structure models: random inclusions versus layers. Homogeneous particles with an effective medium theory are used in the former case, and the layered spheres and core-mantle spheroids are used in the latter one. Calculations for nonspherical layered particles are still rather seldom mainly because Waterman's method does not work well for them; for example, in [12] we have demonstrated that the method can treat just sphere-like particles (layered spheroids with the aspect ratio less than about 2).

In this paper, we extend the electrostatic analysis of applicability of Waterman's method from [11] to layered axisymmetric particles. In Section 2, we formulate the electrostatic problem for layered particles and apply Waterman's method to solve it. We analyze the infinite systems of linear algebraic equations produced by the method and obtain the conditions of its applicability to layered nonspherical particles in Section 3. In Section 4, we consider these conditions in the particular case of layered spheroids, compare our results with available numerical calculations, concern the question on singularities of the analytic continuations of wave fields in presence of a spheroidal particle that is far from being clear and that gave rise to controversial results of the earlier analysis, and finally discuss the applicability of the results obtained to the light scattering case. We draw the conclusions in Section 5.

\section{Basic Equations}

2.1. Electrostatic Problem. We consider this problem for a particle with $J$ layers and introduce the scalar potentials $\Phi(\vec{r})$ related to the fields in the usual way: $\vec{E}=-\nabla \Phi$. Then the problem is reduced to the scalar Helmholtz equation, and, for the $j$ th medium, we have

$$
\Delta \Phi^{(j)}(\vec{r})=0,
$$

where $j=1$ corresponds to the medium outside the particle and $j=J+1$ corresponds to its core.

The boundary conditions at the layer surfaces are

$$
\begin{aligned}
\Phi^{(j)}(\vec{r}) & =\Phi^{(j+1)}(\vec{r}), \\
\frac{\partial \Phi^{(j)}(\vec{r})}{\partial n_{j}}=\epsilon_{j+1} \frac{\partial \Phi^{(j+1)}(\vec{r})}{\partial n_{j}}, & \\
& \vec{r} \in S_{j},
\end{aligned}
$$

where $S_{j}$ is the inner surface of the $j$ th layer, $n_{j}$ is the external normal to $S_{j}, \epsilon_{j+1}=\varepsilon_{j+1} / \varepsilon_{j}$ is the relative permittivity $\varepsilon$ of the $(j+1)$ th medium, and $j=1,2, \ldots, J$.
As usual, we separate all the fields in two parts $\vec{E}^{(j)}=\vec{E}_{1}^{(j)}+$ $\vec{E}_{2}^{(j)}$, so that the corresponding potentials $\Phi_{1}^{(j)}$ are regular at the coordinate origin and $\Phi_{2}^{(j)}$ tend to zero at infinity.

2.2. Solution by Waterman's Method. This method is based on the well-known surface integral equations called the extended boundary condition (see, e.g., [3]). In the electrostatic case, those equations written in terms of the potentials $\Phi^{(j)}$ can be simplified as discussed in [11], and one arrives to a surface integral equation for each $S_{j}$ (see for more details [13]):

$$
\begin{aligned}
& \left(\epsilon_{j+1}-1\right) \\
& \cdot \int_{S_{j}}\left\{\frac{\partial\left(\Phi_{1}^{(j+1)}\left(\vec{r}^{\prime}\right)+\Phi_{2}^{(j+1)}\left(\vec{r}^{\prime}\right)\right)}{\partial n_{j}^{\prime}} G\left(\vec{r}, \vec{r}^{\prime}\right)\right\} \mathrm{d} s^{\prime} \\
& \quad= \begin{cases}\Phi_{1}^{(j)}(\vec{r})-\Phi_{1}^{(j+1)}(\vec{r}), & \vec{r} \in D_{j}, \\
-\Phi_{2}^{(j)}(\vec{r})+\Phi_{2}^{(j+1)}(\vec{r}), & \vec{r} \in R^{3} \backslash \bar{D}_{j},\end{cases}
\end{aligned}
$$

where $D_{j}$ is the domain inside $S_{j}$ and we assume that the potential $\Phi_{1}^{(j)}$, being regular at the coordinate origin, can be continued into the interior region $D_{j+1}$, while $\Phi_{2}^{(j+1)}$, being finite at infinity, can be continued into the outer region $R^{3} \backslash \bar{D}_{j}$.

We expand the Green function $G\left(\vec{r}, \vec{r}^{\prime}\right)$ and all the potentials in terms of the eigenfunctions of the Laplace operator, $\Psi_{m l}^{(i)}(\vec{r})$, being analogs of the spherical wave functions (for more details, see [11]):

$$
\begin{aligned}
& G\left(\vec{r}, \vec{r}^{\prime}\right)= \begin{cases}\sum_{m=0}^{\infty} \sum_{l=m}^{\infty} \Psi_{m l}^{(1)}\left(\vec{r}^{\prime}\right) \Psi_{m l}^{(3)}(\vec{r}) & r>r^{\prime}, \\
\sum_{m=0}^{\infty} \sum_{l=m}^{\infty} \Psi_{m l}^{(1)}(\vec{r}) \Psi_{m l}^{(3)}\left(\vec{r}^{\prime}\right) & r<r^{\prime},\end{cases} \\
& \Phi_{1}^{(j)}(\vec{r})=\sum_{m=0}^{\infty} \sum_{l=m}^{\infty} a_{m l}^{(j)} \Psi_{m l}^{(1)}(\vec{r}), \\
& \Phi_{2}^{(j)}(\vec{r})=\sum_{m=0}^{\infty} \sum_{l=m}^{\infty} b_{m l}^{(j)} \Psi_{m l}^{(3)}(\vec{r}) .
\end{aligned}
$$

Note that when one considers the light scattering by small particles in comparison with the wavelength of the incident radiation, the problem is reduced to the electrostatic one with a uniform applied field (see, e.g., [14]). Hereafter, we will consider this case too.

The main contribution to the scattered field in the farfield zone is then known to be given by a dipole term. So, in the expansions (5) of the corresponding potentials $\Phi_{1}^{(J+1)}$ and $\Phi_{2}^{(J+1)}$, we leave only one term with $l=1$ and $m=0$ or 1 . Note that the orientation of the applied field relative to the particle symmetry axis defines the azimuthal mode, that is, the value of $m$, which is the same for all the expansions (5) as there is the separation of variables relative to the azimuthal angle $\varphi$ (see for more details [15]). 
In the usual way, we get a system of linear algebraic equations relative to the unknown potential expansion coefficients (see, e.g., [13]):

$$
\begin{aligned}
\vec{a}^{(j)}=A_{31}^{(j)} \vec{a}^{(j+1)}+A_{33}^{(j)} \vec{b}^{(j+1)}, \\
\vec{b}^{(j)}=A_{11}^{(j)} \vec{a}^{(j+1)}+A_{13}^{(j)} \vec{b}^{(j+1)}, \\
\text { with } \vec{a}^{(j)}=\left\{a_{m l}^{(j)}\right\}_{l=m}^{\infty}, \vec{b}^{(j)}=\left\{b_{m l}^{(j)}\right\}_{l=m}^{\infty}, \text { and } \\
\left\{A_{31}^{(j)}\right\}_{n l}=\delta_{n l}+\left(\epsilon_{j+1}-1\right) L_{n l, m}^{31, j}, \\
\left\{A_{33}^{(j)}\right\}_{n l}=\left(\epsilon_{j+1}-1\right) L_{n l, m}^{33, j}, \\
\left\{A_{11}^{(j)}\right\}_{n l}=-\left(\epsilon_{j+1}-1\right) L_{n l, m}^{11, j}, \\
\left\{A_{13}^{(j)}\right\}_{n l}=\delta_{n l}-\left(\epsilon_{j+1}-1\right) L_{n l, m}^{13, j},
\end{aligned}
$$

where $\delta_{n l}$ is the Kronecker delta, $j=1,2, \ldots, J$, and $m=0$ or 1. The surface integrals are

$$
L_{n l, m}^{k i, j}=\int_{S_{j}} \Psi_{m n}^{(k)}(\vec{r}) \frac{\partial \Psi_{m l}^{(i)}(\vec{r})}{\partial n_{j}} \mathrm{~d} s .
$$

Using (6), we can relate the coefficients of expansions of the electrostatic analogs of the incident $\left(\vec{a}^{(1)}\right)$ and scattered $\left(\vec{b}^{(1)}\right)$ fields with those of the field in the core $\left(\vec{a}^{(J+1)}\right)$ as follows:

$$
\begin{aligned}
& \vec{a}^{(1)}=A_{1} \vec{a}^{(J+1)}, \\
& \vec{b}^{(1)}=A_{2} \vec{a}^{(J+1)},
\end{aligned}
$$

where $A_{1}$ and $A_{2}$ are some combinations of the matrices $A_{11}^{(j)}, \ldots, A_{33}^{(j)}$ with $j=1,2, \ldots, J$ (see for more details [13]). So, the transition $T$-matrix is $T=A_{2} A_{1}^{-1}$.

Solution of system (9) gives the solution to the problem. In electrostatics, the main characteristic in the far-field zone is polarizability. When the applied field is parallel to the particle symmetry axis $(m=0)$, it is equal to $\alpha_{z}=b_{01}^{(1)} / 3 a_{01}^{(1)}$. When the incident field is perpendicular to the symmetry axis $(m=$ $1)$, we get $\alpha_{x}=b_{11}^{(1)} / 3 a_{11}^{(1)}$.

\section{Analysis}

Here we extend our analysis of Waterman's method, made for homogeneous particles in the electrostatic case in [11], to layered nonspherical particles with the axial symmetry. The analysis is based on consideration of the properties of the infinite system of linear algebraic equations relative to the field expansion coefficients. It is known that such systems have a solution and it can be found by the reduction method if the matrix elements and free terms are limited in a certain way (see for more details [16]). Note that, being different in some details, our analysis is generally identical to that earlier proposed by Kyurkchan and Smirnova and described, for example, in [9].
3.1. Earlier Results for Homogeneous Particles. For such particles, it was shown in [11] that in the electrostatic case the infinite system produced by Waterman's method has the only solution and the solutions to truncated systems of the size $N \times N$ converge to it with growing $N$ being the number of terms kept in the expansions under the condition

$$
\sigma_{1}<\sigma_{2}
$$

where the quantities $\sigma_{1}$ and $\sigma_{2}$ are determined from the asymptotic behavior of the matrix elements, being some surface integrals of wave functions and their derivatives, for large index values and depend only on the particle shape (and a dimension parameter).

The quantities $\sigma_{1}$ and $\sigma_{2}$ can be interpreted as the distances to the farthest and closest singularities of the analytic continuation of the "scattered" and internal fields, respectively, keeping in mind that these quantities and these distances are determined by the same equations [11]. Then condition (10) gets a physical sense that there should be a spherical layer where the expansions of these fields converge.

Note that application of these results to homogeneous spheroids $(J=1)$ needs a correction as for them (and only for them) in electrostatics we have (see for more details [11])

$$
L_{n l, m}^{31, J}=0 \text { for } n>l
$$

that is, the matrix $A_{1}=A_{31}^{(J)}$ of system (9) is triangular. For a uniform applied field, its expansion (5) includes only one term with $l=1$ and $m=0$ or 1 . So, the free terms of the system in the left part of (9) form a vector with only one nonzero element. Then the solution to this system having the triangular matrix will be a vector with only one nonzero element. In other words, we get that the electrostatic field inside a spheroid is to be uniform. This is the well-known feature of spheroids (see, e.g., [17] and references therein). So, in electrostatics, the uniform internal field and equality (11) for the integrals $L_{n l, m}^{31,1}$ which make the matrix triangular are related. Obviously, the infinite system (9) with such a triangular matrix $A_{1}$ always has a unique solution.

Thus, condition (10) defines the applicability range of Waterman's method for homogeneous particles in the electrostatic case, but for spheroids and only for them this condition must be replaced with something like $\sigma_{1}<\infty$, that is, with the conclusion that the method is applicable to any homogeneous spheroids.

3.2. Analysis for Layered Particles. We apply the approach described in [11] to system (6) produced by Waterman's method for a layered particle in the electrostatic case. To use the theorems of [16], we should bring this system to a standard form by the substitution:

$$
\begin{aligned}
& a_{m l}^{(j)}=\frac{\widetilde{a}_{m l}^{(j)}}{\sigma^{l}}, \\
& b_{m l}^{(j)}=\widetilde{b}_{m l}^{(j)} \sigma^{l},
\end{aligned}
$$

where $\sigma$ is a constant. 
Let us start with a core-mantle particle. From (6) and (7), we easily get the system

$$
\begin{aligned}
\tilde{a}_{m n}^{(1)} & =\frac{\epsilon_{2}+1}{2} \widetilde{a}_{m n}^{(2)}+\left(\epsilon_{2}-1\right) \\
\cdot & {\left[\sum_{l=m}^{\infty} \frac{\left(L_{n l, m}^{31,1}-(1 / 2) \delta_{n l}\right)}{\sigma^{l-n}} \widetilde{a}_{m l}^{(2)}+\sum_{l=m}^{\infty} L_{n l, m}^{33,1} \sigma^{l+n} \tilde{b}_{m l}^{(2)}\right], } \\
\tilde{b}_{m n}^{(1)} & =\frac{\epsilon_{2}+1}{2} \widetilde{b}_{m n}^{(2)}-\left(\epsilon_{2}-1\right) \\
\cdot & {\left[\sum_{l=m}^{\infty} \frac{L_{n l, m}^{11,1}}{\sigma^{n+l}} \widetilde{a}_{m l}^{(2)}+\sum_{l=m}^{\infty} \frac{\left(L_{n l, m}^{13,1}+(1 / 2) \delta_{n l}\right)}{\sigma^{n-l}} \widetilde{b}_{m l}^{(2)}\right], } \\
\tilde{a}_{m n}^{(2)} & =\frac{\epsilon_{3}+1}{2} \widetilde{a}_{m n}^{(3)}+\left(\epsilon_{3}-1\right) \sum_{l=m}^{\infty} \frac{\left(L_{n l, m}^{31,2}-(1 / 2) \delta_{n l}\right)}{\sigma^{l-n}} \widetilde{a}_{m l}^{(3)}, \\
\tilde{b}_{m n}^{(2)} & =-\left(\epsilon_{3}-1\right) \sum_{l} \frac{L_{n l, m}^{11,2}}{\sigma^{n+l}} \widetilde{a}_{m l}^{(3)},
\end{aligned}
$$

where we took into account that for large $n$ the integrals $L_{n n, m}^{13, j}$ and $L_{n n, m}^{31, j}$ will tend to $-1 / 2$ and $1 / 2$, respectively.

The asymptotic behavior of the integrals $L_{n l, m}^{i k, j}$ is an important element of the analysis. Follow the estimates of these integrals made by using the saddle-point approximation in [11]; we obtain

$$
\begin{aligned}
& L_{n l, m}^{11, j} \sim\left[\sigma_{1}^{(j)}\right]^{l}, \\
& L_{n l, m}^{13, j} \sim\left[\sigma_{2}^{(j)}\right]^{-l}, \\
& L_{n l, m}^{31, j} \sim\left[\sigma_{1}^{(j)}\right]^{l}, \\
& L_{n l, m}^{33, j} \sim\left[\sigma_{2}^{(j)}\right]^{-l} \\
& L_{n l, m}^{11, j} \sim\left[\sigma_{1}^{(j)}\right]^{n}, \\
& L_{n l, m}^{13, j} \sim\left[\sigma_{1}^{(j)}\right]^{n}, \\
& L_{n l, m}^{31, j} \sim\left[\sigma_{2}^{(j)}\right]^{-n}, \\
& L_{n l, m}^{33, j} \sim\left[\sigma_{2}^{(j)}\right]^{-n}
\end{aligned}
$$$$
\text { for } l \gg n \text {, }
$$

for $l \ll n$,

where $\sigma_{1}^{(j)}$ and $\sigma_{2}^{(j)}$ are the quantities determined from the asymptotic behavior of the surface integrals $L_{n l, m}^{i k, j}$ and depending on the shape of the boundary surface $S_{j}$ and a dimension parameter (see for more details [11] and references therein).
For $n \gg 1$, we have got

$$
\begin{aligned}
& L_{n n, m}^{31, j}=\frac{1}{2}+O\left(\frac{1}{n}\right), \\
& L_{n, m}^{13, j}=-\frac{1}{2}+O\left(\frac{1}{n}\right),
\end{aligned}
$$

and when $l \sim n \gg 1$ and $l \neq n$,

$$
L_{n l, m}^{31, j}=O\left[(n+l)^{-k}\right] \text {, }
$$

where $k$ is any natural. The last estimate is valid under the condition that the function $r^{(j)}(\theta)$ defining the axisymmetric surface $S_{j}$ is infinitely differentiated. In the general case, the speed of the decrease of the integrals depends on smoothness of $r^{(j)}(\theta)$.

The use of these asymptotic formulae allows us to find the conditions under which system (6) has a solution. According to [11], it occurs when the system written in the form

$$
x_{n}=\sum_{l=m}^{\infty} c_{n l} x_{l}+b_{n}, \quad n=1,2, \ldots
$$

is limited so that there is a number $N$ such that for $n<N$

$$
\begin{aligned}
\sum_{l=m}^{\infty}\left|c_{n l}\right| & <\infty \\
\left|b_{n}\right| & <\infty
\end{aligned}
$$

and for $n \geq N$

$$
\begin{gathered}
\sum_{l=m}^{\infty}\left|c_{n l}\right| \leq p<1, \\
\left|b_{n}\right| \leq K
\end{gathered}
$$

where $p$ and $K$ are some constants.

To satisfy conditions (23), the sums in (13)-(16) must be asymptotically finite. So, considering the asymptotic behavior of the matrix elements of (13), we see that the first and second sums in that equation are limitedly provided, respectively:

$$
\begin{aligned}
& \sum_{l=m}^{\infty}\left|\frac{\sigma_{1}^{(1)}}{\sigma}\right|^{l} \leq \tilde{p}_{1}, \\
& \sum_{l=m}^{\infty}\left|\frac{\sigma}{\sigma_{2}^{(1)}}\right|^{l} \leq \tilde{p}_{2},
\end{aligned}
$$

where $\widetilde{p}_{1}$ and $\widetilde{p}_{2}$ are some constants. It can take place only when $\sigma_{1}^{(1)}<\sigma$ and $\sigma<\sigma_{2}^{(1)}$, and hence we get the conditions

$$
\begin{aligned}
\sigma_{1}^{(1)} & <\sigma, \\
\sigma & <\sigma_{2}^{(1)} .
\end{aligned}
$$

It is easy to see that the same conditions are derived from (14). In a similar way, from (15) and (16), we get more conditions:

$$
\begin{aligned}
\sigma_{1}^{(2)} & <\sigma, \\
\sigma & <\sigma_{2}^{(2)},
\end{aligned}
$$

where $\sigma$ is the same constant as in (25). 
Our accurate consideration of the restrictions (23) for system (13)-(16) has naturally shown that (25) and (26) are the necessary and sufficient conditions under which for coremantle particles system (6) satisfies the restrictions and hence has a solution that can be found by the reduction method.

It is not difficult to prove the obvious general statement that for particles with $J$ layers these conditions can be written as

$$
\max \left\{\sigma_{1}^{(j)}\right\}<\min \left\{\sigma_{2}^{(j)}\right\}
$$

where $j=1,2, \ldots, J$.

Thus, condition (27) is a generalization of condition (10) to layered particles in electrostatics, except for the case of spheroids when this condition must be modified according to the ideas of [11]. Note that the addition of any one layer can just make the applicability range of the method more narrow.

\section{Discussion}

The spheroidal shape is the most often used one in modeling of nonspherical scatterers. Here we discuss the modification of condition (27) for such models and compare our results with available results of numerical calculations in both the electrostatic and light scattering cases.

4.1. The Case of Layered Spheroids. Following the consideration of homogeneous spheroids in [11], it is easy to demonstrate that for the core of a layered spheroidal particle in the electrostatic case

$$
L_{n l, m}^{31, J}=0 \text { for } n>l .
$$

This changes the method applicability condition (27) but not as strongly as for homogeneous spheroids. It is easy to see that we just have to skip a part of the condition related to $\sigma_{2}^{(J)}$. So, we get

$$
\begin{aligned}
& \max \left\{\sigma_{1}^{(1)}, \sigma_{1}^{(2)}, \ldots, \sigma_{1}^{(J)}\right\} \\
& \quad<\min \left\{\sigma_{2}^{(1)}, \sigma_{2}^{(2)}, \ldots, \sigma_{2}^{(J-1)}\right\} .
\end{aligned}
$$

The physical sense of this condition may be the fact that there should be a spherical layer, where the expansions of all the fields, except for that in the core, converge.

In the particular case of spheroids with the confocal surfaces of the layers, we have $\sigma_{1}^{(j)}=d^{(j)} / 2=d^{(1)} / 2$, where $d^{(j)}$ is the focal distance of the surface $S_{j}$ and $j=1,2, \ldots, J$. Then in the electrostatic case we derive the condition

$$
\sigma_{1}^{(1)}<\min \left\{\sigma_{2}^{(1)}, \sigma_{2}^{(2)}, \ldots, \sigma_{2}^{(J-1)}\right\} .
$$

Thus, while Waterman's method is applicable to any homogeneous spheroids, the method can give results only for a rather narrow set of even core-mantle spheroids and an addition of any next layer can just further decrease the method's applicability range.

4.2. Confocal Core-Mantle Spheroids. These particles present a very important particular case when there is a well-known analytical solution to the electrostatic problem (see, e.g., [14]). So, we can understand some important details.

Our analysis says that the method is applicable under condition (30) that takes here $(J=2)$ the form

$$
\sigma_{1}^{(1)}<\sigma_{2}^{(1)}
$$

where the quantities $\sigma_{1}^{(1)}$ and $\sigma_{2}^{(1)}$ are related to the particle surface. For a confocal core-mantle particle with the semiaxes $a$ and $b$, we have $\sigma_{1}^{(1)}=d^{(1)} / 2=\sqrt{a^{2}-b^{2}}$ and $\sigma_{2}^{(1)}=$ $2 a b / d^{(1)}=2 a b / \sqrt{a^{2}-b^{2}}$, which gives the condition

$$
\frac{a}{b}<\sqrt{2}+1
$$

This electrostatic problem has always the solution that allows one directly to calculate the polarizability [14], but this can be done by Waterman's method only under condition (31). In [8] we have considered in detail what happens, when condition (31) is not satisfied and hereafter just remind the main points. It is easy to see that system (9) has the matrix

$$
A_{1}=A_{31}^{(1)} A_{31}^{(2)}+A_{33}^{(1)} A_{11}^{(2)}
$$

where for spheroids the product of the triangular matrices including the integrals $L_{n l, m}^{31, j}$ is triangular (the regular part of the field in the layer is obviously uniform and hence $L_{n l, m}^{31,1}=0$ for $n>l$ ) and does not affect the solution existence analysis. Problems are connected with the term $A_{33}^{(1)} A_{11}^{(2)}$. They appear as follows.

It is not difficult to obtain the polarizability from the solution by Waterman's method; for example, in the case of $m=0$,

$$
\alpha_{z}=\frac{V}{4 \pi} \frac{\left(\epsilon_{2}-1\right)\left[1+\left(\epsilon_{3}-1\right) L_{z}^{2}\right]+\left(\epsilon_{3}-1\right)\left[1-\left(\epsilon_{2}-1\right)\left(L_{z}^{1}-1\right)\right]\left(V_{2} / V_{1}\right)}{\left[1+\left(\epsilon_{2}-1\right) L_{z}^{1}\right]\left[1+\left(\epsilon_{3}-1\right) L_{z}^{2}\right]-\left(\epsilon_{2}-1\right)\left(\epsilon_{3}-1\right) \sum_{l=1}^{\infty} L_{1 l, m}^{33,1} L_{1 l, m}^{11,2}},
$$

where $L_{z}^{j}$ is the usual geometric factor of the surface $S_{j}$ along $z$-axis [14] and $V_{j}$ is the volume of the domain $D_{j}$; that is, the particle volume $V=V_{1}$.
Relation (34) coincides with the expression given in [14], provided that the series converges as follows (see for details [13]): 


$$
\sum_{l=1}^{\infty} L_{1 l, m}^{33,1} L_{1 l, m}^{11,2}=L_{z}^{1}\left(L_{z}^{1}-1\right) \frac{V_{2}}{V_{1}} .
$$

From the asymptotic behavior of the integrals (17), we easily find that the sum cannot converge if $\sigma_{1}^{(2)}=\sigma_{1}^{(1)} \geq \sigma_{2}^{(1)}$.

We have proven that this series converges (to the given value) under condition (31) by using special computations made with accuracy of about 300 digits due to the use of the Multiple Precision Arithmetic Library and some other packages in [8]. For instance, we found that the ratio of 402nd term to 400 th one was equal to 1.0 with accuracy better than $10^{-30}$ just for $a / b=\sqrt{2}+1$.

Thus, using the case of confocal core-mantle spheroids, we completely confirmed our condition of applicability of Waterman's method in electrostatics and saw in detail what happens when the method's applicability condition is not satisfied: the solution of the problem exists but cannot be found by the method because of the divergence of a series.

4.3. Problem of Singularities of the Field inside Spheroids. The problem is as follows. When in electrostatics we consider a homogeneous spheroid with the semiaxes $a$ and $b$, we formally get the quantities $\sigma_{1}$ and $\sigma_{2}$ from the asymptotic behavior of the matrix elements (see for more details [11]). The values of $\sigma_{1}=\sqrt{a^{2}-b^{2}}$ and $\sigma_{2}=2 a b / \sqrt{a^{2}-b^{2}}$, where $a$ and $b$ are the spheroid semiaxes, are determined by the same equations as the distances to singularities of the analytic continuations of the scattered and internal field analogs, respectively. However, when the applied electrostatic field is uniform, the field inside the spheroid is uniform too. This produces a contradiction on one side; we formally have got the value of $\sigma_{2}$ that has the sense of the distance to singularities of the internal field, but, on the other side, the analytic continuation of this uniform field should not have any singularities.

Some authors believe that, for a spheroidal shape, $\sigma_{2}$ is not related to any singularity of wave fields (and often formally put $\sigma_{2}=\infty$ ). Then, for homogeneous spheroids, they get the applicability condition $\sigma_{1}<\infty$, which agrees with our conclusion that the method is applicable to any spheroid.

Our analysis of confocal core-mantle spheroids shows that the situation is not as simple. We have firmly established the applicability condition $\sigma_{1}^{(1)}=\sigma_{1}^{(2)}<\sigma_{2}^{(1)}$, where $\sigma_{2}^{(1)}=2 a b / \sqrt{a^{2}-b^{2}}$ (see Section 4.1). This condition does not include $\sigma_{2}^{(2)}$, and one may consider $\sigma_{2}^{(2)}=\infty$. However, in electrostatics for the uniform applied field and a confocal core-mantle spheroid, the field in its mantle is known to be uniform too [14]. So, the situation becomes even less clear than for the homogeneous spheroids; the applicability condition now includes $\sigma_{2}^{(1)}$ related to the distance to singularities of the field inside the mantle, while this field is uniform.

Some understanding of this problem is given by socalled inverse spheroids: axisymmetric particles obtained from homogeneous spheroids with the profile $r=r_{\mathrm{sph}}(\theta)$ by their inversion $r(\theta)=a b / r_{\mathrm{sph}}(\theta)$. In [11] we have considered such particles in electrostatics and found singularities of the analytic continuations of the scattered and internal field analogs at the distances $\widetilde{\sigma}_{1}=\sqrt{a^{2}-b^{2}} / 2$ and $\widetilde{\sigma}_{2}=a b / \sqrt{a^{2}-b^{2}}$, respectively. After the back inversion to spheroids, the singularities of the internal field transformed into those of the scattered field in foci at the distance $a b / \widetilde{\sigma}_{2}=$ $\sqrt{a^{2}-b^{2}}$, while the singularities of the scattered field should become those of the internal field at $a b / \widetilde{\sigma}_{1}=2 a b / \sqrt{a^{2}-b^{2}}$. Thus, we see that the singularities of the field inside the inverse spheroids transform correctly, while the singularities of the scattered field that must become those of the field inside the spheroid just disappear. We believe that this point needs a further analysis.

4.4. Applicability to the Light Scattering Case. The light scattering problem is known to be reduced to the electrostatic one when the size of a scatterer is small in comparison with the wavelength of the incident radiation (see for details [14]). However, the transition from light scattering to electrostatics is not as simple as it seems because of the differences in the potential definitions and the radial functions. Waterman needed certain efforts to show this transition in his paper [5].

Despite these differences, the similarity of the electrostatic and light scattering cases is strong enough because of the resemblance of the wave functions, of the wave fields in the presence of a particle, of their expansions, and of singularities of their analytic continuations (see for more details [11]). That is why in [5] Waterman recommended using the electrostatic case in analyzing the light scattering methods, and we extend the results of our analysis to light scattering.

This extension is supported by the agreement of our electrostatic conclusions with the theoretical works and numerical calculations made in light scattering case.

Earlier analyses of the method in this case are discussed in detail in [11], and an agreement with our results is found everywhere. For example, in [9], the authors gave condition (10) of light scattering applicability of their pattern equation method which is a version of Waterman's method formulated just for the far-field zone.

Concerning the numerical tests, the electrostatic condition (10) gives very clear predictions, for example, for the Chebyshev (axisymmetric) particles having the profile $r(\theta)=$ $r_{0}(1+\varepsilon \cos n \theta)$. Our light scattering calculations [15] (as well as our recent special electrostatic ones for inverse spheroids) well agree with these predictions. The exceptional case of homogeneous spheroids that we describe in Section 3.1 and in more detail in [11] is confirmed by very accurate light scattering computations in [10].

It is not simple to compare our electrostatic results even for layered spheroids with numerical light scattering calculations based on Waterman's method as they should not have been published because of their unexpectedly narrow range of parameter values. In [12], we developed a code based on this method and applied it to three-layered spheroids. We could not find well converging solutions for prolate particles with the layers of an equal volume and of the same aspect ratio $a / b$ already for $a / b \gtrsim 1.7$. Note that condition (29) is satisfied 
for such particles for $a / b \leqslant 2.2$. Thus, we see that our results generally agree with these numerical tests.

\section{Conclusions}

In the electrostatic case, we have obtained the general condition of Waterman's method applicability to layered scatterers, which depends only on the shape of their layers. We have considered this condition in the particular case of layered spheroids and noted that the set of such particles that can be treated by Waterman's method is surprisingly small, which has a large practical value. Using the explicit solution for confocal core-mantle spheroids, we consider what happens when the condition is not satisfied. We have found that the conclusions of our analysis agree with the results of different numerical calculations. A consideration of coremantle spheroids has revealed another aspect of the "virtual singularities" of the analytic continuation of the field inside a homogeneous spheroid which still requires a better understanding.

The similarity of the electrostatic and light scattering cases, earlier theoretical works on homogeneous scatterers (see for a review [11]), and the agreement of our conclusions with the numerical calculations available allow us to suggest that the results obtained are valid for light scattering as well.

\section{Competing Interests}

The authors declare that there are no competing interests regarding the publication of this paper.

\section{Acknowledgments}

The work was partly supported by the Ministry of Education and Science of Russia within the state assignment for SUAI, the research grants of St. Petersburg Univ. (6.38.18.2014), and RFBR (16-02-00194 and 16-52-45005).

\section{References}

[1] P. C. Waterman, "Matrix formulation of electromagnetic scattering," Proceedings of the IEEE, vol. 53, no. 8, pp. 805-812, 1965.

[2] P. C. Waterman, "Scattering by dielectric obstacles," Alta Frequenza, vol. 38, pp. 348-352, 1969.

[3] M. I. Mishchenko, L. D. Travis, and A. A. Lacis, Scattering, Absorption, and Emission of Light by Small Particles, Cambridge University Press, 2002.

[4] M. I. Mishchenko, N. T. Zakharova, N. G. Khlebtsov, G. Videen, and T. Wriedt, "Comprehensive thematic T-matrix reference database: a 2014-2015 update," Journal of Quantitative Spectroscopy and Radiative Transfer, vol. 178, pp. 276-283, 2016.

[5] P. C. Waterman, "Matrix methods in potential theory and electromagnetic scattering," Journal of Applied Physics, vol. 50, no. 7, pp. 4550-4566, 1979.

[6] T. Rother and M. Kahnert, Electromagnetic wave scattering on nonspherical particles, vol. 145 of Springer Series in Optical Sciences, Springer, Heidelberg, Germany, 2nd edition, 2014.
[7] M. I. Mishchenko and L. D. Travis, "T-matrix computations of light scattering by large spheroidal particles," Optics Communications, vol. 109, no. 1-2, pp. 16-21, 1994.

[8] V. G. Farafonov and V. B. Il'in, "On the applicability of a spherical basis for spheroidal layered scatterers," Optics and Spectroscopy, vol. 115, no. 5, pp. 745-752, 2013.

[9] A. G. Kyurkchan and N. I. Smirnova, Mathematical Modeling in Diffraction Theory Based on a Priori Information on the Analytical Properties of the Solution, Elsevier, 2015.

[10] W. R. C. Somerville, B. Auguié, and E. C. Le Ru, "Accurate and convergent T-matrix calculations of light scattering by spheroids," Journal of Quantitative Spectroscopy and Radiative Transfer, vol. 160, pp. 29-35, 2015.

[11] V. Farafonov, V. Il'in, V. Ustimov, and M. Prokopjeva, "On the analysis of Waterman's approach in the electrostatic case," Journal of Quantitative Spectroscopy and Radiative Transfer, vol. 178, pp. 176-191, 2016.

[12] V. G. Farafonov, B. V. Il'in, and M. S. Prokopjeva, "Light scattering by multilayered nonspherical particles: a set of methods," Journal of Quantitative Spectroscopy and Radiative Transfer, vol. 79-80, pp. 599-626, 2003.

[13] V. G. Farafonov and M. V. Sokolovskaja, "Construction of the Rayleigh approximation for axisymmetric multilayered particles, making use of eigenfunctions of the Laplace operator," Journal of Mathematical Sciences, vol. 194, pp. 104-116, 2013.

[14] C. F. Bohren and D. R. Huffman, Absorption and Scattering of Light by Small Particles, Wiley, Hoboken, NJ, USA, 1983.

[15] V. G. Farafonov and V. B. Il'in, Light Scattering Reviews, Springer, 2006.

[16] L. V. Kantorovich and V. I. Krylov, Approximate Methods of Higher Analysis, Interscience, 1958.

[17] H. Kang and G. W. Milton, "Solutions to the Pólya-szegö conjecture and the weak Eshelby conjecture," Archive for Rational Mechanics and Analysis, vol. 188, no. 1, pp. 93-116, 2008. 


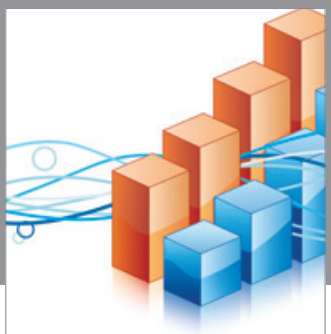

Advances in

Operations Research

vatem alat4

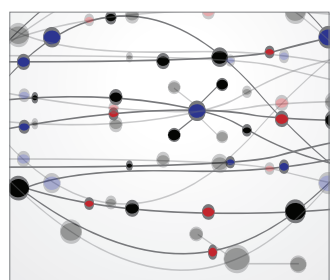

\section{The Scientific} World Journal
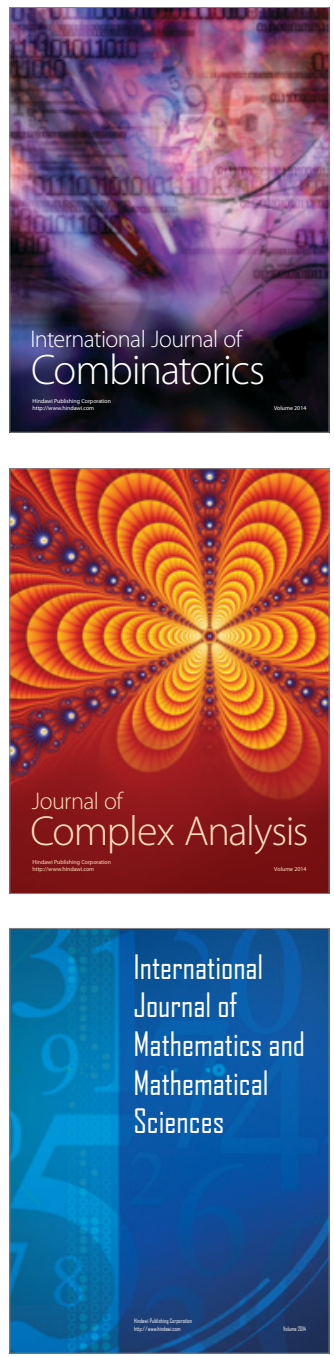
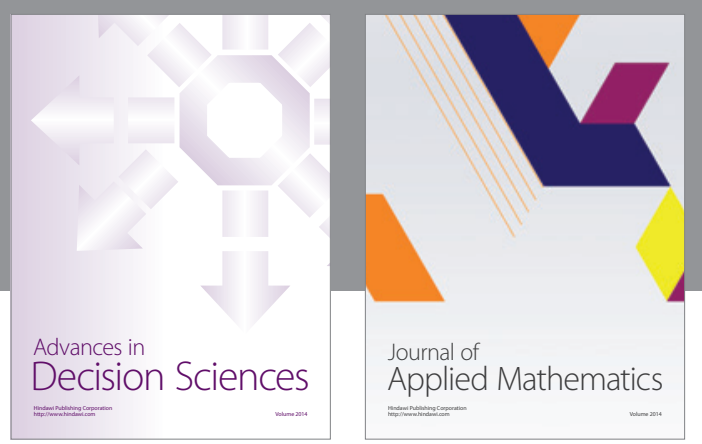

Algebra

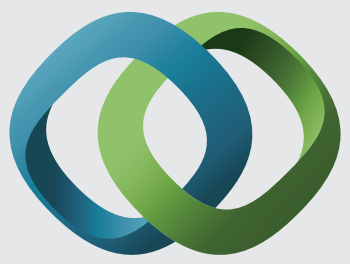

\section{Hindawi}

Submit your manuscripts at

https://www.hindawi.com
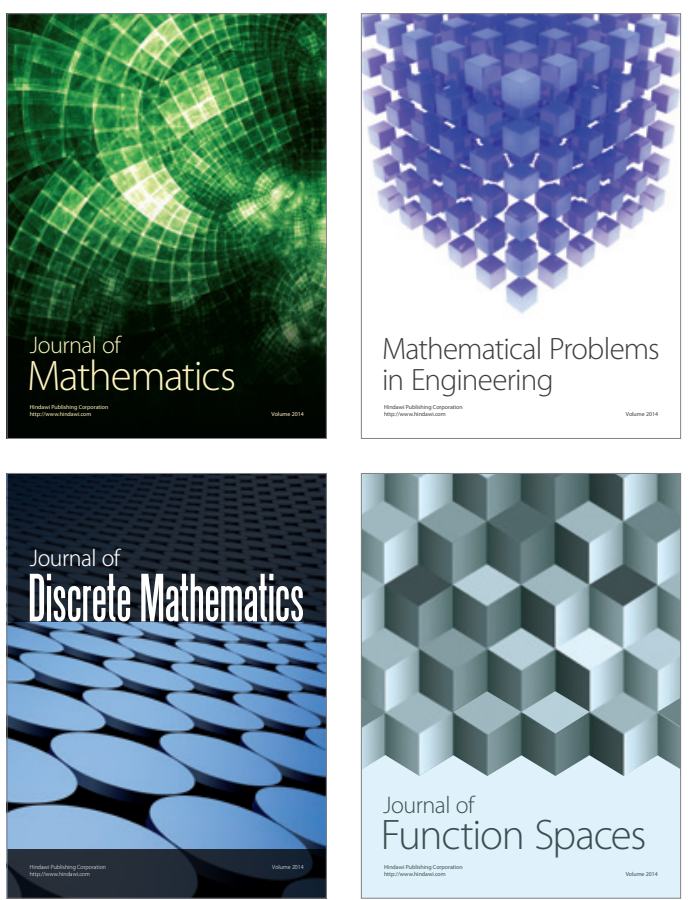

Mathematical Problems in Engineering
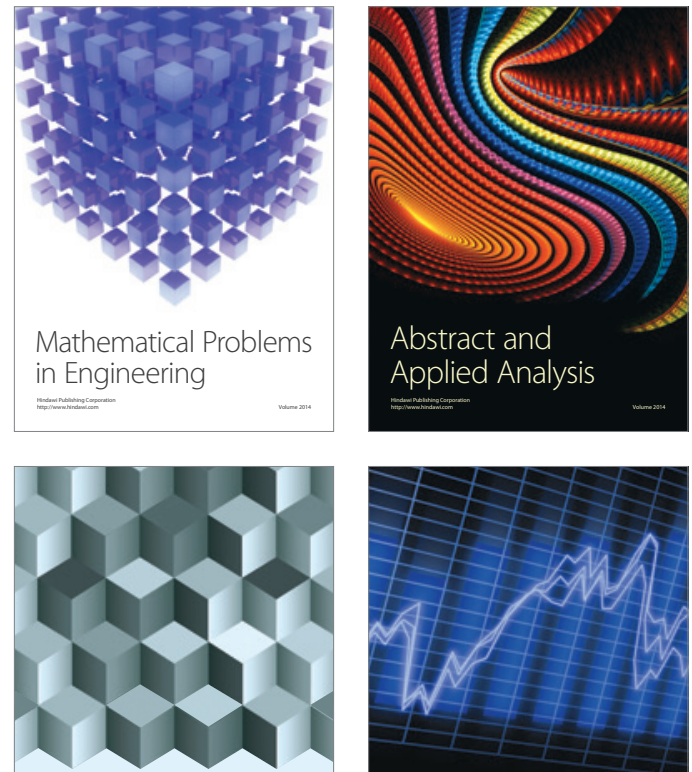

Journal of

Function Spaces

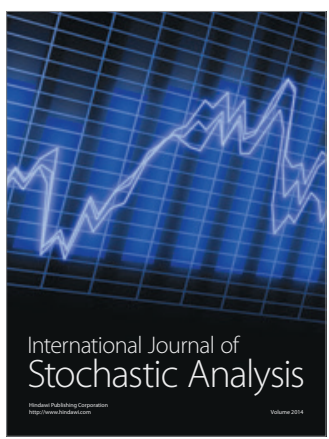

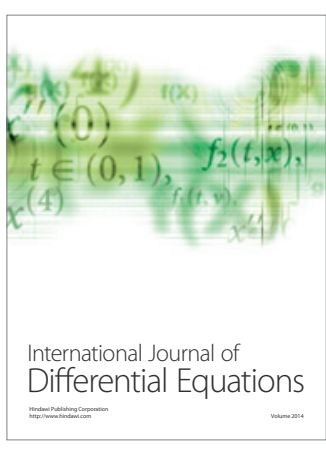
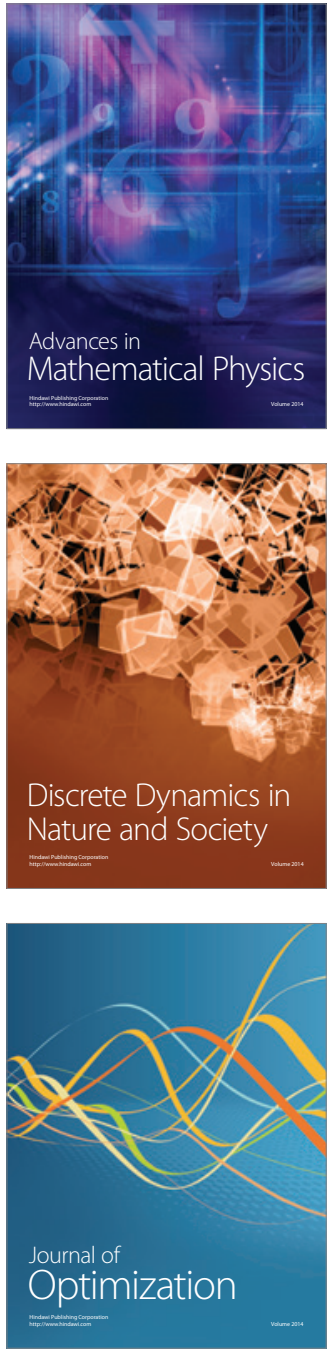